REVIEWS

\title{
Quality of life in dementia
}

\author{
Jan Motley * Carrie Buch \\ School of Nursing, Oakland University, Rochester, Michigan, USA
}

Received: January 25, 2016

DOI: $10.5430 /$ jnep.v6n9p122
Accepted: February 21, $2016 \quad$ Online Published: May 23, 2016

URL: http://dx.doi.org/10.5430/jnep.v6n9p122

\begin{abstract}
This paper discusses the concept of quality of life as it relates to people with dementia. Dementia is a disease that causes a decline in functional abilities which leads to a decrease in quality of life. Quality of life can be defined as an overall well-being that combines objective and subjective evaluations of an individual's perceived state of satisfaction in their abilities and achievements. Wellbeing is further categorized in six dimensions which are: physical wellbeing, material wellbeing, emotional wellbeing, social wellbeing, spiritual wellbeing, and mental wellbeing. If these six dimensions of wellbeing are not achieved in the life of an individual with dementia, they may experience a decline in overall quality of life. Nurses have a critical role in helping persons with dementia maintain or improve their QOL by gaining a better understanding of QOL, its significance, and what it means to each individual. In this paper, strategies for maintaining or improving quality of life in the six dimensions of wellbeing are discussed. In addition, areas of future research are recommended.
\end{abstract}

Key Words: Quality of life, Wellbeing, Dementia

\section{INTRODUCTION}

Dementia is a devastating disease that profoundly affects the quality of life (QOL) of individuals and their families. It includes multiple cognitive and physical deficits that occur gradually over time. These progressive changes will significantly affect the individual's QOL. Presently, there is no cure for this disease, so it is important to help individuals with dementia maintain the best QOL possible as their symptoms worsen. Nurses have a critical role in helping those with dementia maintain their QOL. In order to be effective in this role, nurses must understand the concept of QOL and how it is affected by dementia. Additionally, it is important to understand dementia and the signs and symptoms of the disease from early to the advanced stage. Therefore, the purpose of this concept analysis is to explore the concept of quality of life and how people with dementia experience it.

\section{WHAT IS THE CONCEPT OF QUALITY OF LIFE?}

In order to have a better understanding of QOL, it is important first to look at the history of QOL and then discuss the definition and dimensions of QOL. It is not always clearly understood what the term quality of life means. This term dates back to the time of Aristotle, ${ }^{[1]}$ a philosopher who wrote about "living well and "the good life" and how it could be supported by public policy. ${ }^{[2]}$ In 1889 , the term QOL was used in a statement by another philosopher, Seth, who believed that QOL was just as important as important as quantity of life. ${ }^{[2]}$

Quality of life concern in nursing came about in the 1850's when Florence Nightingale evaluated the care her nurses delivered and tried to improve areas that were below standards for those times. ${ }^{[3]}$ In 1930, researchers began to have more interest in the term QOL and made several attempts to

*Correspondence: Jan Motley; Email: jmotley@oakland.edu; Address: School of Nursing, Oakland University, Rochester, Michigan, USA. 
investigate, measure, and define this concept. ${ }^{[3]}$ The Medline database introduced the subject heading QOL in 1975, while it was introduced by Index Medicus in 1977, and, finally, by the Cumulative Index for Nursing and Allied Health (CINAHL) in 1983. ${ }^{[4]}$

Furthermore, Advances in Nursing Science dedicated an entire issue to QOL in 1985 thus emphasizing the importance of QOL in nursing. This issue focused on explaining the concept of QOL from different perspectives such as understanding the concept in nursing, developing an instrument to measure QOL, QOL as an outcome measure in nursing research, the idea that producing and maintaining a state of pleasure is a nursing goal, an examination of immigrant women, and self-esteem later in life. ${ }^{[4]}$ Within the last three decades, advances in health care have led to an increased interest in QOL and healthcare systems are seeking ways to improve QOL. Therefore, it is critical for nurses to understand quality of life and the different dimensions that make up this important concept.

Quality of life can be defined as an overall well-being that combines objective and subjective evaluations of an individual's perceived state of satisfaction in their abilities and achievements. This concept consists of six dimensions that further describe the elements that are needed to enhance an individual's QOL. These dimensions are physical wellbeing, material wellbeing, social wellbeing, emotional wellbeing, spiritual wellbeing, and mental wellbeing. ${ }^{[5,6]}$

The first dimension, physical well-being, is the ability to function normally in basic activities of daily living such as bathing, walking, dressing, eating, or overall functional mobility. ${ }^{[6]}$ The second dimension, material wellbeing, evaluates an individual's financial stability, standards of living, financial security, and overall possessions. ${ }^{[5]}$ Satisfaction in financial security may contribute to a good QOL for some people. This dimension is critical to an individual's QOL because those with higher income can more easily buy food and other necessities. ${ }^{[7]}$

The third dimension, social wellbeing, relates to the person's ability to participate in society through family gatherings, friendship, employment, school activities, volunteering, etc. ${ }^{[6]}$ The fourth dimension of QOL, emotional wellbeing, is based on how a person may feel about themselves, their ability to manage life's challenges and the quality of their relationships. ${ }^{[8]}$ Emotional health is the ability to adapt to change, have a sense of purpose, a feeling of completeness and contentment, and the ability to create fulfilling relationships, etc. ${ }^{[8]}$

The next dimension, spiritual wellbeing, is a sense of peace

Published by Sciedu Press and contentment coming from an individual's relationship with the spiritual aspects of life. ${ }^{[9]}$ It is also understood as being concerned with what has meaning and value in life, which goes beyond human boundaries, promotes a connection with one's self, God/higher power, others and the environment such as nature. ${ }^{[10]}$ Some of the QOL enhancing benefits of spiritual wellbeing include: feeling content with life, maintaining balance and control of life, building positive relationships, feeling of having a purpose and meaning in life, and experiencing a connection with a power greater than oneself. ${ }^{[11]}$

The last dimension, mental wellbeing, implies that cognitive abilities are intact, and there are no feelings of depression, anxiety, or any negative emotions. ${ }^{[6]}$ Physical, material, social, emotional, spiritual, and mental wellbeing are all needed to have an enhanced wellbeing or QOL. When human needs are met, and a person can meaningfully pursue their goals, a satisfied QOL is achieved; however, this accomplishment is very challenging for people with dementia.

\section{QUALITY OF LIFE IN DEMENTIA}

The QOL of a person with dementia should be the main focus of care. It is important for care providers to respond to the needs, wishes, and values of people with dementia. Dementia causes a decline in a person's physical abilities. Because dementia affects areas of the brain that are responsible for movement and balance, a person with dementia may have an increasing difficulty maintaining a steady gait therefore causing a risk for safety. ${ }^{[12]}$ This decline in physical mobility and balance is an important determinant of a person's independence and physical wellbeing. A decline or loss of mobility can lead to more physical complications such as constipation that often results from immobility. ${ }^{[12]}$

Often the person with dementia becomes frustrated because of their decreased level of physical functioning. ${ }^{[13]}$ Other physical problems that may occur in dementia include eating problems as the person with dementia could have difficulty swallowing or chewing resulting in significant weight loss. Control of bowel or bladder may also be affected. ${ }^{[12]}$ It is not uncommon for a person with dementia to have problems with physical functioning. ${ }^{[14]}$ The main focus is their safety, along with their dignity and wellbeing. Some solutions may be as simple as keeping their living space free from clutter to avoid falls and injuries, making sure they are given the opportunity to go to the bathroom every two hours or as needed to avoid incontinence, and decreasing noise and stimulation to decrease their anxiety or aggression. ${ }^{[14]}$ In addition, for the person with dementia who has swallowing difficulties, it is important to cut their food into bite size pieces or even puree meals along with thickened fluids to avoid weight loss and 
dehydration. ${ }^{[14]}$ These declines in functional abilities such as difficulty remembering things, thinking clearly, communicating with others, mood swings, changes in personality and behavior, may decrease a person's QOL by preventing the person with dementia from living an independent life style which can lead to a decline in physical wellbeing. ${ }^{[15]}$

Furthermore, persons with dementia may express dissatisfaction with their financial situation or material well-being. Since they are no longer able to work because of the progress of the disease, they may not be able to afford basic goods such as food or have adequate living accommodations. ${ }^{[7]}$ During this time, people with dementia can find support in community and social services that are available in their area. These services include Meals on Wheels, adult day programs, volunteer visits, and support from their church. Family members or caregivers may also want to help the person with dementia to budget their money to avoid superfluous spending and misplacing money. ${ }^{[16]}$

People with dementia may also suffer from social isolation because they are not able to continue with their previously held roles, or those roles may have been taken away from them. They are no longer able to contribute and help take care of their families or communities, despite their desire to remain dedicated and involved, therefore decreasing their social well-being and QOL. ${ }^{[17]}$ To help the person with dementia maintain a good QOL, they need to have a sense of purpose so they are at a reduced risk of becoming affected by social isolation. Encourage them to join clubs that share their interest such as book clubs, and hobbies that promote social interaction, for example, a knitting club. ${ }^{[18]}$ Giving the person with dementia the responsibility of nurturing a plant or pet has been known to have a positive impact on the QOL of the person with dementia. ${ }^{[18]}$ Pet owners are less likely to suffer from loneliness or depression, and are more secure and motivated. ${ }^{[18]}$

Because of dementia, people may experience feelings of anger, frustration, or confusion, and have no control over their emotions. As a result, they are unsure of themselves around family, friends, or in social situations. Persons with dementia may also experience changes in mood or become short-tempered. These behaviors are a direct result of the disease process and decrease emotional well-being. ${ }^{[19]}$

Some ways to help people with dementia enhance their emotional wellbeing include maintaining close relationships with family and friends and allowing them to experience a wide range of emotions. It is important to help them understand that there is no right or wrong way to feel. ${ }^{[19]}$ Additionally, discussing feelings and emotions with family, friends, a counselor, doctor, or religious leader such as a pastor can improve emotional wellbeing. Relaxation techniques such as meditation or prayer can also help enhance emotional wellbeing. Furthermore, simple exercises such as walking, stretching, yoga, and deep breathing may improve emotional wellbeing and QOL. ${ }^{[7]}$

A person with dementia may display disruptive behaviors that can separate them from the rest of the community and limit their opportunities to worship with others. ${ }^{[20]}$ Limiting the opportunity to worship with others can cause a decrease in spiritual wellbeing. Persons with dementia use spirituality as a coping method to enhance QOL and cope with such stressors as loss of independence, role and identity, feelings of vulnerability and loss of the ability to communicate. ${ }^{[10]}$

People with mild to moderate dementia, who maintained a strong religious faith were content and had hope in their life because of their belief in God or another deity. ${ }^{[21]}$ People with end-stage dementia who lack the cognitive capacity or ability to make decisions about their care such as taking part in religious servicers or Holy Communion, could have that decision made for them as a part of their best interest and wellbeing. This decision could be made by a family member or care provider. ${ }^{[21]}$

Furthermore, dementia causes a decline in an individual's mental abilities which leads to confusion and repetitive behavior. Often, the person with dementia is aware of these changes in cognition and may become irritable, agitated, or even tearful. ${ }^{[22]}$ For example, a 65-year-old woman who has been accustomed to daily walks suddenly forgets her way back home. Forgetting everyday routines can be very distressing for the person with dementia which causes the individual to become fearful of getting lost and leaving their home to enjoy leisure activities. This behavior of isolation can cause a decrease in mental well-being and QOL.

To decrease these behaviors in people with dementia, try to distract the person with meaningful activities such as light housework to decrease restlessness. If the person with dementia displays repeated actions or repeating words, distract them with simple activities such as folding laundry or polishing furniture. If the person with dementia becomes physically or verbally aggressive, be calm and reassuring, look for an immediate cause, give the person space to calm down, and leave the area if it is unsafe. ${ }^{[23]}$

To prevent the person with dementia from wandering away from home unattended with the risk of becoming lost, distract the person with another activity, move the locks on the outside doors out of reach, and put reminders such as coats and hats out of sight and reach. If the person with dementia becomes suspicious and thinks others are trying to hurt them 
or steal their possessions, don't argue or try to reason, don't take accusations personally, provide comfort and distract with another activity. ${ }^{[23]}$

As the disease progresses, the person with dementia may lose their abilities and functions they consider important to their QOL. While the symptoms are mild to moderate, the person with dementia is aware of what contributes to their pleasure and well-being. As the disease progresses to its later stages, the person with dementia will require help adapting to their changing abilities and participating in meaningful activities. ${ }^{[24]}$ Once these individuals are no longer able to make decisions for themselves, they will need the help of caregivers, family members, and healthcare providers to provide care that will enhance QOL for the person with dementia. ${ }^{[24]}$

\section{NURSING IMPLICATIONS}

Through research, it is known that dementia decreases QOL because of the cognitive and functional decline. ${ }^{[12,14]}$ QOL may be perceived differently by each person with dementia because every person has a different perspective of what they feel is important to them. Some health care providers who provide care for people with dementia do not understand the cognitive and functional changes that are related to dementia. ${ }^{[24]}$

Nurses have the ability to assist persons with dementia and help them to maintain a good QOL by gaining a better understanding of QOL, its significance, and what it means to each individual. Nurses can educate family and caregivers on the early detection and symptoms of dementia in order to provide adequate treatment and planning as the disease progresses. $^{[25]}$

Nurses can also collaborate with interdisciplinary team members to work together to maintain continuity of care which could enhance the QOL for persons with dementia. In addition, nurses and health care workers may want to consider learning how to communicate with the person with dementia, such as understanding how facial expressions may convey emotions such as sadness or joy, observing which activities are known to give the person with dementia pleasure, and giving the person with dementia opportunities to make choices. These opportunities will increase independence and enhance QOL. ${ }^{[24]}$

Furthermore, it is important for nurses to monitor the overall health of the person with dementia and ensure that appropriate treatments are given. Failure to treat other illnesses such as a urinary tract infection can worsen the symptoms of dementia. Moreover, nurses should make sure that everyone who provides care for the person with dementia understands that persons with the disease should be valued and treated as individuals. $^{[24]}$

\section{Conclusion}

Nurses and other members of the healthcare team who have a better understanding of the concept of QOL will implement a plan of care based on the goals and needs of the person with dementia. Individualized care and activities are ways to improve QOL for persons with dementia by decreasing depression, loneliness, and behavioral disturbances. If healthcare workers are to increase their understanding of QOL and how it is experienced by persons with dementia, future research is needed to examine this concept thoroughly. This research should focus on improving care within the dimensions of QOL for people with dementia. Moreover, there is a minimal amount of literature that discusses QOL from the perspective of the person with dementia. Most of the literature on this topic focuses on the caregiver's perspective of QOL of the person with dementia. Therefore, future research should focus on measuring QOL from the perspective of the person with dementia as well as ways to enhance and preserve QOL for these individuals as it is a progressive disease that affects all areas of QOL.

\section{CONFLiCTS OF INTEREST Disclosure}

The authors declare that there is no conflict of interest.

\section{REFERENCES}

[1] Ettema PT. The concept of quality of life in dementia in the different stages of the disease. International Psychogeriatrics. 2005; 17(3): 353-370. http://dx.doi.org/10.1017/S1041610205002073

[2] Forward S. State of the art report on life quality assessment in the field of transport and mobility. 2003. Available from: http: //www.factum.at/asi/download/ASI_D21_final.pdf

[3] Theofanidis D, Fountouki A. Quality of life and nursing: A position paper. International Journal of Caring Sciences. 2010; 3(2): 56-62. Available from: http://www. internationaljournalof caring

Published by Sciedu Press
sciences.org/docs/Vol3_Issue2_02_Theofanidis.pdf

[4] Padilla GV, Grant MM, Ferrell B. Nursing research into quality of life. Quality of Life Research. 1992; 1(5): 341-348. http: //dx.doi.org/10.1007/BF00434948

[5] Felce D, Perry J. Quality of life: Its definition and measurement. Research in Developmental Disabilities. 1995; 16(1): 51-74. http: //dx.doi.org/10.1016/0891-4222(94)00028-8

[6] National Institute of Dental and Craniofacial Research. 2014. Effects on well-being and quality of life. Available from: http: //www.nidcr.nih.gov/DataStatistics/SurgeonGeneral/ 
sgr/chap6.htm?_ga=1.58203178.1018805056.1430434196

[7] Sirgy M. Material well-being. The psychology of human life (pp 325-351). 2012. Available from: http://link.springer.com/ book/10.1007\%2F978-94-007-4405-9

[8] U.S. Department of Health \& Human Services. (n.d.). Feeling great starts here. Available from: http://www.foh.hhs.gov/Calend ar/august.html

[9] Jonas: Mosby's Dictionary of Complementary and Alternative Medicine. 2015. Spiritual well-being. Available from: http://medical-dictionary.thefreedictionary.com/sp iritual+well-being

[10] Dalby P, Sperlinger DJ, Boddington S. The lived experience of spirituality and dementia in older people living with mild to moderate dementia. Dementia. 2011; 11(1): 75-94. http://dx.doi .org/1 $0.1177 / 1471301211416608$

[11] Aston University. 2015. Spiritual wellbeing. Available from: http://www.aston.ac.uk/staff/hr/wellbeing/psychol ogical-wellbeing/spiritual-wellbeing/

[12] Dementia Guide. 2015b. Symptom library. Available from: http://www.dementiaguide.com/symptomlibrary/physi calchanges/balance/

[13] Dementia Guide. 2015a. About dementia. Available from: http: //www.dementiaguide.com/aboutdementia/

[14] Lift Simplified Caregiving. 2011. The physical effects of dementia. Available from: https://www.liftcaregiving.com/article s/single/the-physical-effects-of-dementia/

[15] Family Caregiver Alliance. 2015. Caregivers guide to understanding dementia behaviors. Available from: https://caregiver.org/ caregivers-guide-understanding-dementia-behaviors

[16] Alzheimer Society Canada. 2014a. Living alone. Available from: http://www.alzheimer.ca/en/Living-with-dementi a/Staying-connected/Living-alone
[17] Duane F, Brasher K, Koch S. Living alone with dementia. Dementia 2011; 12(1): 123-136. http://dx.doi.org/10.1177/1471301 211420331

[18] Dixon J. How to prevent social isolation in the elderly. 2013 Available from: http://www.carltonseniorliving.com/ho w-to-prevent-social-isolation-in-the-elderly/

[19] Alzheimer's Association. 2015. Taking care of yourself. Available from: http://www.alz.org/i-have-alz/taking-car e-of-yourself.asp

[20] Powell C. Dementia and caring: Remembering spiritual needs. 2015. Available from: http://enrichmentjournal.ag.org/201401/ 201401_028_Dementia.cfm

[21] Higgins P. The spiritual and religious needs of people with dementia. 2011. Available from: http://www.cmq.org.uk/CMQ/2011/04 -spiritual-needs-dementia.html

[22] Mental Health Foundation. (n.d.). Mental health in later life. Available from: http://www.mentalhealth.org.uk/help-infor mation/mental-health-a-z/0/older-people/

[23] Alzheimer Society Canada. 2014c. Understanding behaviour. Available from: http://www.alzheimer.ca/en/Living-with-dem entia/Understanding-behaviour

[24] Alzheimer Society Canada. 2014b. Quality of life. Available from: http://www.alzheimer.ca/en/Living-with-dementi a/Caring-for-someone/Quality-of-life

[25] Rose MK, Lopez RP. Transitions in dementia care: Theoretical support for nursing roles. 2012. Available from: http://nursingworld.org/MainMenuCategories/ANAMar ketplace/ANAPeriodicals/0JIN/TableofContents/Vol-1 7-2012/No2-May-2012/Transitions-in-Dementia-Care. html\#Recommendations 